\title{
Smoothing Tensor-Valued Images Using Anisotropic Geodesic Diffusion
}

\author{
Fan Zhang and Edwin R. Hancock \\ Department of Computer Science, University of York, \\ York, YO10 5DD, UK \\ $\{z f a n$, erh $\}$ @s. york.ac.uk
}

\begin{abstract}
This paper considers the feature space of DT-MRI as a differential manifold with an affine-invariant metric. We generalise Di Zenzo's structure tensor to tensor-valued images for edge detection. To improve the quality of the edges, we develop a generalised Perona-Malik method for smoothing tensor images. We demonstrate our algorithm on both synthetic and real DT-MRI data.
\end{abstract}

\section{Introduction}

Diffusion tensor magnetic resonance imaging (DT-MRI) 1] endows each voxel a $3 \times 3$ symmetric positive-definite matrix, which measures the anisotropic behaviour of water diffusion in the white matter of the brain. The feature space of DT-MRI data is no longer a linear space, but a curved convex half-cone in $R^{n^{2}}$. Thus edge or interface [2] detection, which is important for segmentation and registration, is more complicated than in scalar-valued or vector-valued images. In an attempt to overcome these difficulties Feddern et al [3] generalise Di Zenzo's 4 concept of structure tensors to tensor-valued images for levelset motions. Using the same structure tensor, O'Donnell, et al 2 introduced a more sophisticated gradient estimation method for DT-MRI edge detection. The structure tensor used simply considers diffusion tensors as vectors in $R^{n^{2}}$. However, it neglects the constraints between components induced by symmetry and positive-definiteness of the tensor.

In this paper we consider the space of diffusion tensors as a differential manifold with an affine invariant metric. In this way we generalise the Di Zenzo's structure tensor to tensor-valued images. In order to reduce the influence of noise and obtain a high quality edge detector, we show how to extend the Perona and Malik [5] anisotropic diffusion method to tensor-valued images. To do this we make use of the exponential map of the tensor data and use geodesic marching. The idea of using a manifold of diffusion tensors, has been recently used to analyse the principle geodesic modes [6] of tensor data and the segmentation of DT-MRI 7]. Pennec et al [8 has developed a framework for the analysis of statistical data residing on manifolds, and has generalised the operations of interpolation, isotropic and anisotropic regularisation for DT-MRI. 


\section{Space of Diffusion Tensors}

Let $\Sigma(r)$ be the set of $r \times r$ real matrices and $G L(r)$ be its subset of non-singular matrices which is a Lie group. Recall that in $\Sigma(r)$ the Euclidean inner product, which is known as the Frobenius inner product, is defined as $\langle A, B\rangle_{F}=\operatorname{tr}\left(A^{T} B\right)$, where $\operatorname{tr}(\cdot)$ denotes the trace and superscript $T$ denotes the transpose.

For a matrix $A$ whose eigen-decomposition is $A=U D U^{T}$, the exponential of $A$ is given by convergent series

$$
\exp A=\sum_{k=0}^{\infty} \frac{1}{k !} A^{k}=U \exp (D) U^{T},
$$

and the inverse logarithm of $A$ is given by

$$
\log A=-\sum_{k=1}^{\infty} \frac{(I-A)^{k}}{k}=U \log (D) U^{T},
$$

where $I$ is the identity matrix.

Let $S(r)$ be the space of $r \times r$ symmetric matrices and $S^{+}(r)$ be the space of symmetric positive-definite matrices. Thus, the feature space $M$ of DT-MRI is identified with $S^{+}(3)$. Through the identity mapping

$$
\psi: P \in S^{+}(r) \rightarrow\left(\sigma_{11}, \ldots, \sigma_{i j}\right), \quad i \leq j, \quad i, j=1, \ldots, r
$$

$S^{+}(r)$ is isomorphic with an open subset $U$ of the $R^{m}$ where $m=\frac{1}{2} r(r+1)$. Thus we could consider $S^{+}(r)$ as a $m$-dimensional differential manifold with $(U, \psi)$ as the coordinate system. At each point $P \in S^{+}(r)$ the tangent space $T_{P} S^{+}(r)$ is equal to $S(r)$. So a basis of $T_{P} S^{+}(r)$ can be defined as

$$
\frac{\partial}{\partial \sigma_{i j}} \leftrightarrow E_{i j} \in S(r), \quad i \leq j, \quad i, j=1, \ldots, r,
$$

and

$$
E_{i j}= \begin{cases}1_{i i} & \text { if } i=j \\ 1_{i j}+1_{j i} & \text { if } i \neq j\end{cases}
$$

where $1_{i j}$ means the $r \times r$ matrix with a 1 at element $(i, j)$ and 0 elsewhere.

We can turn $S^{+}(r)$ into a Riemannian manifold by introducing a Riemannian metric $g$ at $P$

$$
g\left(\frac{\partial}{\partial \sigma_{i j}}, \frac{\partial}{\partial \sigma_{k l}}\right)=g\left(E_{i j}, E_{k l}\right)=\operatorname{tr}\left(P^{-1} E_{i j} P^{-1} E_{k l}\right) .
$$

This is the same as the positive-definite inner product used by 698 , i.e., $\langle A, B\rangle_{P}=\operatorname{tr}\left(P^{-1} A P^{-1} B\right), A, B \in T_{P} S^{+}(r)$, which is invariant under group actions of $G L(r)$. 
Thus, for a smooth curve $C:[a, b] \rightarrow S^{+}(r)$ in $S^{+}(r)$, the length of $C(t)$ can be computed via the invariant metric

$$
\ell(C)=\int_{a}^{b}\left\|C^{\prime}(t)\right\|_{C(t)}=\int_{a}^{b} \sqrt{\operatorname{tr}\left(C(t)^{-1} C^{\prime}(t)\right)^{2}},
$$

which is also invariant under $G L(r)$, i.e., $C(t) \mapsto G C(t) G^{T}, G \in G L(r)$. The distance between two points $A, B \in S^{+}(r)$ is the infinum of lengths of curves connecting them, i.e.,

$$
d(x, y):=\underset{C}{\operatorname{argmin}}\{\ell(C) \mid C(a)=A, C(b)=B\} .
$$

The curve satisfying this infinum condition is a geodesic. In $S^{+}(r)$ the geodesic with initial point at $I$ and tangent vector $W \in T_{I} S^{+}(r)$ given by $\exp (t W)$. Using invariance under group action $G L(r)$, an arbitrary geodesic $\Gamma(t)$ such that $\Gamma(0)=P$ and $\Gamma^{\prime}(0)=W$ is given by

$$
\Gamma_{(P, W)}(t)=P^{\frac{1}{2}} \exp \left(t P^{-\frac{1}{2}} W P^{-\frac{1}{2}}\right) P^{\frac{1}{2}} .
$$

Thus, the geodesic distance between two points $A$ and $B$ in $S^{+}(r)$ is

$$
d(A, B)=\left\|\log \left(A^{-1} B\right)\right\|_{F}=\sqrt{\sum_{i=1}^{n}\left(\log \lambda_{i}\right)^{2}},
$$

where $\lambda_{i}$ are the eigenvalues of $A^{-1} B$.

We can relate an open subset of the tangent space $T_{P} S^{+}(r)$ to a local neighbourhood of $P$ in $S^{+}(r)$ using the exponential map Exp : $\Omega \subset T_{P} S^{+}(r) \rightarrow$ $S^{+}(r)$, which is defined as $\operatorname{Exp}_{P}(W)=\gamma_{(P, W)}(1)$. Geometrically, $\operatorname{Exp}_{P}(W)$ is a point of $S^{+}(r)$ obtained by marking out a length equal to $|W|$ commencing from $P$, along a geodesic which passes through $P$ with velocity equal to $\frac{W}{|W|}$. From Equation 9, it follows that

$$
\exp _{P}(W)=P^{\frac{1}{2}} \exp \left(P^{-\frac{1}{2}} W P^{-\frac{1}{2}}\right) P^{\frac{1}{2}} .
$$

Since $\exp _{P}$ is a local diffeomorphism, it has an inverse map, the so-called logarithmic map $\log _{P}: S^{+}(r) \rightarrow B_{\epsilon}(0) \subset T_{P} S^{+}(r)$ where $\log _{P}\left(\gamma_{(P, W)}(t)\right)=t W$. Thus, for a point $A$ near $P$ it also follows

$$
\log _{P}(A)=P^{\frac{1}{2}} \log \left(P^{-\frac{1}{2}} A P^{-\frac{1}{2}}\right) P^{\frac{1}{2}} .
$$

\section{Generalised Structure Tensor}

For tensor-valued images, the image features live on a $m$-dimensional manifold $M=S^{+}(r), m=\frac{1}{2} r(r+1) \quad(r=3$ for DT-MRI), which we call the feature space or feature manifold. An image is a map from a domain $\Omega$ to $M$, i.e., $f: \Omega \in R^{n} \rightarrow M$, where $n=2$ for planar images and $n=3$ for volume images. 
Drawing on ideas from Di Zenzo's pioneering work [4], we can generalise the structure tensor to tensor-valued images. At each point $x=\left(x_{1}, \ldots, x_{n}\right) \in \Omega$ we wish to find the direction with maximal variations in the image. For two points $x=\left(x_{1}, \ldots, x_{n}\right)$ and $x^{\prime}=\left(x_{1}^{\prime}, \ldots, x_{n}^{\prime}\right)$, the difference of vector image values is the $f\left(x^{\prime}\right)-f(x)$. As the distance between the two points $\left\|x^{\prime}-x\right\|$ becomes infinitesimal, the local variation $d f$ of the image values is given by

$$
d f=\sum_{i=1}^{n} \frac{\partial f}{\partial x_{i}} d x_{i} .
$$

Then the square vector norm is

$$
d f^{2}=\sum_{i=1}^{n} \sum_{j=1}^{n}\left(\frac{\partial f}{\partial x_{i}} \cdot \frac{\partial f}{\partial x_{j}}\right) d x_{i} d x_{j},
$$

where $\frac{\partial f}{\partial x_{i}}$ is the directional derivative of $f$ along $x_{i}$. If we define $h$ as $h_{i j}:=$ $\frac{\partial f}{\partial x_{i}} \cdot \frac{\partial f}{\partial x_{j}}, i, j=1, \ldots, n$, we have the following quadratic form

$$
d f^{2}=d x^{T} h d x, \text { where } d x=\left(d x_{1}, \ldots, d x_{n}\right)^{T} .
$$

When $m \geq n$, we can consider an image $f$ as a $n$-dimensional manifold $H$ embedded in $M$, i.e., $\Phi: H \rightarrow M$. Then $\frac{\partial f}{\partial x_{i}}, i=1, \ldots, n$ is a basis of the tangent space $T_{x} H$ at $x$. Thus, the quadratic form in Equation [15] is the first fundamental form of the manifold $H[104$. It also follows that $h$ is the metric tensor of $H$, which is symmetric and semi-positive definite. The quantity $h$ is sometimes called the structure tensor in image processing. We note that a similar idea of considering an image as a surface embedded in a space-feature space has been used in [11] for scalar and color image smoothing.

For tensor-valued images, since the feature space is not Euclidean $R^{m}$ but a curved manifold $M$ with Riemannian metric $g$, we can not calculate the metric $h$ of $H$ directly. Since we have already investigated the space of $M$ in Section 2 and introduced the metric $g$ of $M$, we can overcome this problem by inducing the metric tensor $h$ of $H$ from the embedding $\Phi: H \rightarrow M$. Let $x_{1}, \ldots, x_{n}$ be the local coordinates of $H$, than the embedding map is

$$
\begin{aligned}
& \left(x_{1}, \ldots, x_{n}\right) \rightarrow\left\{\Phi_{1}=\sigma_{11}\left(x_{1}, \ldots, x_{n}\right), \ldots, \Phi_{k}=\right. \\
& \left.\sigma_{i j}\left(x_{1}, \ldots, x_{n}\right), \ldots, \Phi_{m}=\sigma_{r r}\left(x_{1}, \ldots, x_{n}\right)\right\}
\end{aligned}
$$

where $i \leq j$. Since the metric tensor $h$ measures the element length of arc $d s_{H}$ in $H$ as

$$
d s_{H}^{2}=\sum_{k=1}^{n} \sum_{l=1}^{n} h_{k l} d x_{k} d x_{l} .
$$

Similarly, for the metric tensor $g$ on the manifold $M$ we have

$$
d s_{M}^{2}=\sum_{i=1}^{n} \sum_{j=1}^{n} g_{i j} d \Phi_{i} d \Phi_{j} .
$$


The embedding $\Phi$ is isometric, which means that the element length appearing in Equation [17 and 18 are equal. Using the rule of change of coordinates $d \Phi_{i}=$ $\frac{\partial \Phi_{i}}{\partial x_{k}} d x_{k}$, the induced Riemannian metric tensor $h$ on $H$ is

$$
h_{k l}=\sum_{i=1}^{n} \sum_{j=1}^{n} g_{i j} \frac{\partial \Phi_{i}}{\partial x_{k}} \frac{\partial \Phi_{j}}{\partial x_{l}} .
$$

The metric tensor (or structure tensor) $h$ of $H$ characterises the local geometry of images. The maximum (or minimum) change of $f$ is in the direction $v$ $=\left(d x_{1}, \ldots, d x_{2}\right),\|v\|=1$ that maximizes or minimizes the quadratic form $d f^{2}$ in Equation 14. The maximum $\lambda_{+}$and minimum $\lambda_{-}$eigenvalues of the structure tensor $h$ give the maximum and mininum rate of changs of $f$ at a given point. Their corresponding eigenvectors $\theta_{+}, \theta_{-}$are the directions of maximum and minimum changes.

For planar images where $n=2, \lambda_{ \pm}, \theta_{ \pm}$are given by

$$
\begin{aligned}
& \lambda_{ \pm}=\frac{h_{11}+h_{22} \pm \sqrt{\left(h_{11}-h_{22}\right)^{2}+4 h_{12}^{2}}}{2} \\
& \theta_{ \pm}=\left(2 h_{12}, h_{22}-h_{11} \pm \sqrt{\left(h_{11}-h_{22}\right)^{2}+4 h_{12}^{2}}\right)^{T} .
\end{aligned}
$$

When the image is scalar-valued, $\theta_{+}=\frac{\nabla I}{\|\nabla I\|}, \quad \theta_{-}=\frac{\nabla I^{T}}{\|\nabla I\|}, \quad \lambda_{+}=\|\nabla I\|^{2}$, $\lambda_{-}=0$. Thus, the gradient is always perpendicular to the edges for scalar images because $\lambda_{-}=0$. However, for multi-valued images, such as color images and tensor-valued images, we also need to consider the minimum rate of change $\lambda_{-}$. It is the values of $\lambda_{+}$together with $\lambda_{-}$that discriminate different local geometries. If $\lambda_{+} \approx \lambda_{-} \approx 0$, the image changes at an equal rate in all directions, so the image surface is almost flat at this point. Thus there are no edges or corners here. If $\lambda_{+} \approx \lambda_{-}>>0$, there is a saddle point of the image surface, and the corresponding point is a corner. If $\lambda_{+}>>\lambda_{-}$, there is step and the corresponding point is an edge. Let $N$ be the gradient norm used to detect edges and corners in images. Three different combinations exist in the literature [12], i.e., $N_{1}=\lambda_{+}$[10], $N_{2}=\sqrt{\lambda_{+}-\lambda_{-}}$[13], and $N_{3}=\sqrt{\lambda_{+}+\lambda_{-}}=\sqrt{\operatorname{tr}(h)}$ [141516]. The combination $N_{1}$ neglects $\lambda_{-}$, and thus is the case in gray-scale images. The combination $N_{2}$ can not detect corners where $\lambda_{+} \approx \lambda_{-}>>0$. The combination $N_{3}$ can be used to detect both edges and corners. For volume images where $n=3$, we could use either $N_{1}$ or $N_{3}$ for edge detection.

\section{Anisotropic Diffusion}

In order to obtain high quality edges, it is necessary to smooth noise before performing edge detection. In [5], Perona and Malik reported an edge preserving smoothing method using anisotropic diffusion. They use anisotropic diffusion equation to evolve gray-scale images $f(x, y): \Omega \subset R^{2} \rightarrow R$

$$
\frac{\partial f}{\partial t}=\operatorname{div}(\rho(\|\nabla f\|) \nabla f),
$$


where $\rho=e^{-\frac{\|\nabla f\|^{2}}{k}}$ or $\rho=\frac{1}{1+\frac{\|\nabla f\|^{2}}{k}}$. Their idea is to halt the heat-flow process at object boundaries. To do this they control the diffusivity using the magnitude of the image gradient. When the gradient is large, which indicates the existence of a likely edge, the value of diffusivity is small. When the gradient is small, on the other hand, the value of diffusivity is large. This method has been improved by using more sophisticated diffusion flows [17. Here, we generalise the PeronaMalik method to tensor-valued images.

The Perona-Malik method discretises Equation 21 on a square lattice and uses the numerical scheme

$$
\begin{aligned}
f_{i, j}^{t+1}= & f_{i, j}^{t}+\lambda\left[\rho_{x_{+}}\left(f_{i-1, j}-f_{i, j}\right)+\rho_{x_{-}}\left(f_{i+1, j}-f_{i, j}\right)\right. \\
& \left.+\rho_{y_{+}}\left(f_{i, j-1}-f_{i, j}\right)+\rho_{y_{-}}\left(f_{i, j+1}-f_{i, j}\right)\right]_{i, j}^{t} .
\end{aligned}
$$

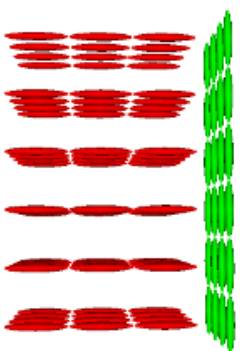

(a1)

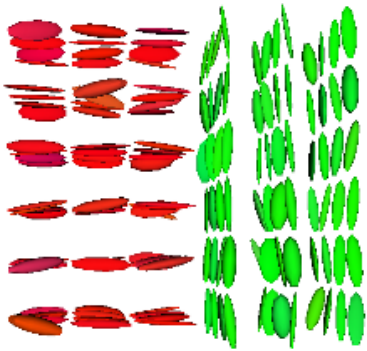

(b1)

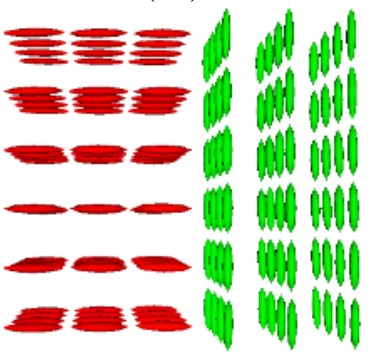

(c1)

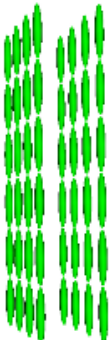

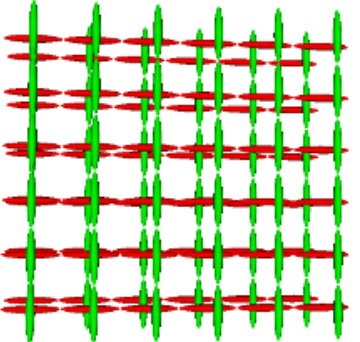

(a2)

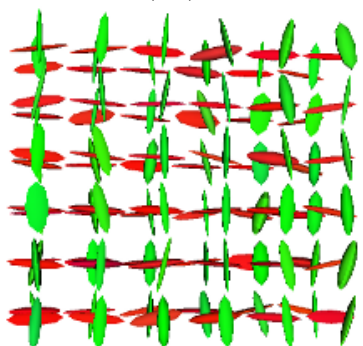

(b2)

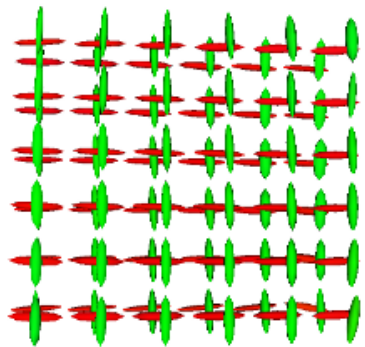

(c2)

Fig. 1. (a1) and (a2): Synthetic tensor fields. (b1) and (b2): Corresponding noisy tensor fields. (c1) and (c2): Filtered results using generalised Perona-Malik anisotropic diffusion with $\lambda=0.25, k=1,5$ iterations. 
For tensor-valued images, since the feature space $M$ is curved, we should use the intrinsic subtraction $\oplus$ and addition $\ominus$ operators on $M[8$ for the purposes of numerical implementation. That is, we let the image values $f(x, y)$ at the location $(x, y)$ diffuse by marching along the geodesics emanating from this location. For two points $A, B$ on $M$, we define $A \oplus B=\operatorname{Exp}_{A}(B)$ and $A \ominus B=\log _{A}(B)$. For tensor-valued images, we have the following numerical scheme

$$
\begin{aligned}
f_{i, j}^{t+1}= & \operatorname{Exp}_{f_{i, j}^{t}}\left\{\lambda \left[\rho_{x_{+}} \log _{f_{i, j}}\left(f_{i-1, j}\right)\right.\right. \\
& +\rho_{x_{-}} \log _{f_{i, j}}\left(f_{i+1, j}\right)+\rho_{y_{+}} \log _{f_{i, j}}\left(f_{i, j-1}\right) \\
& \left.\left.+\rho_{y_{-}} \log _{f_{i, j}}\left(f_{i, j+1}\right)\right]_{i, j}^{t}\right\}
\end{aligned}
$$

where $\rho_{x_{+}}=\exp \left(-\left\|\log _{f_{i, j}}\left(f_{i-1, j}\right)\right\|^{2} / k\right)$ and similar definition for others.

\section{Experiments}

We have applied our Riemannian edge detector and the generalised Perona-Malik anisotropic diffusion to synthetic and real-world tensor-valued images.

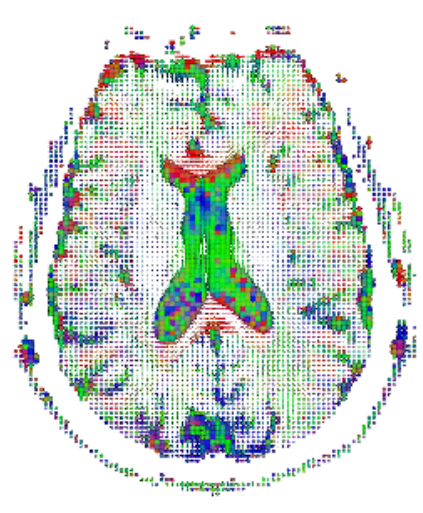

a) A slice of DT-MRI

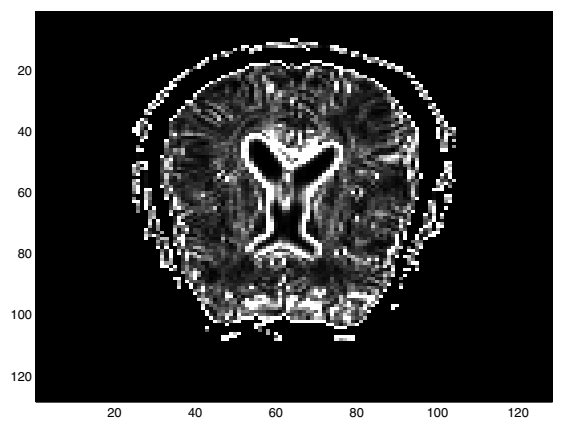

c) Riemannian structure tensor

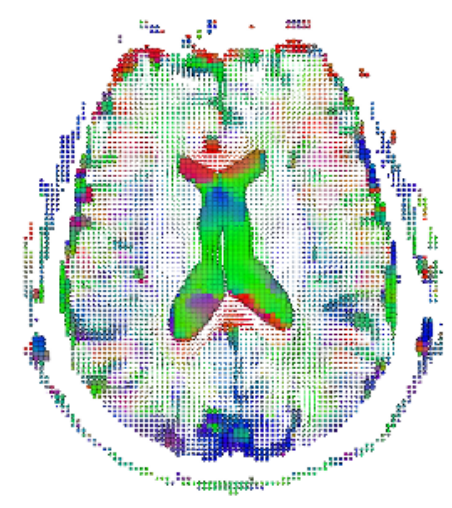

b) Filtered result

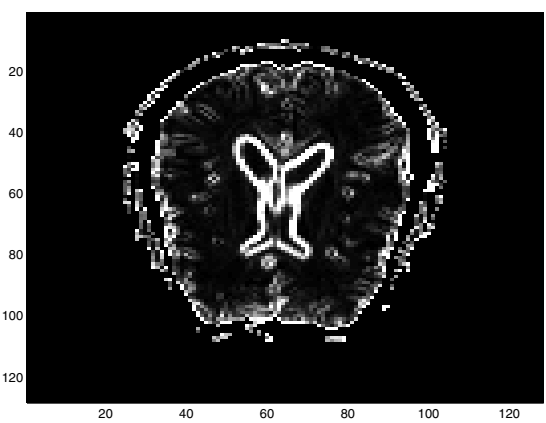

d) Euclidean structure tensor

Fig. 2. Real DT-MRI example 
Fig. 1 shows the results of the generalised anisotropic diffusion on two synthetic noisy tenor fields. We first generate two noise-free tensor fields with different complexity. Field (a1) is fairly simple, while (a2) contains crossing fibers. We corrupt the tensor fields by adding the same quantity of independent and identically distributed (IID) additive noise to eigenvectors and eigenvalues of the tensors respectively. We then apply our algorithm to regularise the noisy tensor fields (b1) and (b2), and the results are shown in (c1) and (c2). The resulted fields show that the generalised anisotropic diffusion well preserves the interfaces between regions and recovers the fine details of the structures, whilst smoothing out the noise.

We have also tested our method on a real-world DT-MRI volume. Fig 2 shows the results of a sample slice. Subfigure (a) is the tensor image visualised using ellipsoids. (b) is the filtered result after applying the anisotropic diffusion. (c) and (d) are the trace of our Riemannian structure tensor and the Euclidean structure tensor 32 of the filtered slice respectively. The results shows that the Riemannian structure tensor is more sensitive for edge detection and can detect the fibres inside the image.

\section{Conclusions}

In this paper we have introduced the structure tensor and the anisotropic diffusion to tensor-valued images. We consider images as surfaces embedded in the space of tensors, which is a differential manifold with an affine-invariant metric. The structure tensor is then the same as the metric tensor of the image surface. Anisotropic diffusion is generalised for tensor-valued images using the exponential map and geodesic marching. Experiments shows that the generalised anisotropic diffusion is efficient to eliminate noise, and our Riemannian structure tensor is more sensitive for edge detection than the Euclidean one.

\section{References}

1. P.J. Baser, J. Mattiello and D. LeBihan : Mr diffusion tensor spectroscopy and imaging. Biophysical Journal 66 (1994) 259-267

2. L. O'Donnell, W. Grimson, C.-F. Westin : Interface detection in diffusion tensor mri. In: Proceedings of MICCAI 2004. (2004) 360-367

3. C. Feddern, J. Weickert, B. Burgeth : Level-set methods for tensor-valued images. (In: Proc. IEEE Workshop on Variational, Geometric and Level Set Methods in Computer Vision) 65-72

4. S. Di Zenzo : A note on the gradient of a multi-image. Computer Vision, Graphics, and Image Processing 33 (1986) 116-125

5. Perona, P., Malik, J.: Scale-space and edge dectection using anisotropic diffusion. IEEE Trans. Pattern Anal. and Machine Intell. 12(7) (1990) 629-639

6. P. Fletcher and S. Joshi : Principal geodesic analysis on symmetric spaces: Statistics of diffusion tensors. (In: Proc. Computer Vision Approaches to Medical Image Analysis, ECCV Workshop (2004)) 
7. C. Lenglet, M. Rousson, R. Deriche and O. Faugeras : A riemannian approach to diffusion tensor images segmentation. In: Proceedings of IPMI 2005. (2005) 591-602

8. X. Pennec, P. Fillard, N. Ayache : A riemannian framework for tensor computing. International Journal of Computer Vision 66(1) (2006) 41-66

9. M. Moakher : A differential geometric approach to the geometric mean of symmetric positive-definite matrices. SIAM Journal on Matrix Analysis and Applications 26(3) (2005) 735-747

10. A. Cumani : Edge detection in multispectral images. CVGIP: Graphical Models and Image Processing 53(1) (1991) 40-51

11. N. Sochen, R. Kimmel and R. Malladi: A general framework for low level vision. IEEE Trans. on Image Processing 7(3) (1998) 310-318

12. Tschumperle, D.: PDE's Based Regularization of Multivalued Images and Applications. PHD Thesis, University of Nice-Sophia Antipolis (2002)

13. G. Sapiro and D.L. Ringach : Anisotropic diffusion of multivalued images with application to color filtering. IEEE Trans. on Image Processing 5(11) (1996) 15821586

14. P. Blomgren and T.F. Chan, : Color tv: Total variation methods for restoration of vector-valued images. IEEE Trans. on Image Processing 7(3) (1998) 304-309

15. B. Tang, G. Sapiro AND V. Caselles : Diffusion of general data on non-flat manifolds via harmonic maps theory: The direction diffusion case. International Journal of Computer Vision 36(2) (2000) 149-161

16. A. Pardo and G. Sapiro : Vector probability diffusion. IEEE Signal Processing Letters $(\mathbf{8}(4))$

17. F. Catte, P. L. Lions, J. M. Morel and T. Coll : Image selective smoothing and edge detection by nonlinear diffusion. SIAM J. Num. Anal. 29 (1992) 182-193 